doi: http://dx.doi.org/10.7124/bc.0009AB

\section{Reorganization of chromatin architecture in Drosophila melanogaster Lamin B mutants}

Semen Bondarenko, Igor V. Sharakhov

Department of Entomology, Virginia Polytechnic Institute and State University, Blacksburg, VA igor@vt.edu

Lamins are essential proteins of the nuclear envelope in animals including insects. Like humans, fruit flies have two types of lamins: B-type and A-type lamins [1]. How the lamins affect three-dimensional chromatin organization is poorly understood. In this study we asked three specific questions: (i) Do dysfunctions of Lamin $B$ caused by the point frameshift mutations lead to detachments of chromatin from the nuclear periphery? (ii) Do the lamin mutations affect the reorganization of the global chromatin architecture and nuclear compartments? (iii) Are any effects of the lamin dysfunction on nuclear architecture cell type-specific? Methods: We used Drosophila melanogaster Lam[K2] and Lam[A25] (BDSC, \#25093, \#25092) homozygotes as lamin-deficiency models. The B-type lamin and the fibrillar component of the nucleolus were visualized using 3D immunostaining by specific antibodies (ADL 67.10, DSHB; ab5821, Abcam). Series of z-stack images of nuclei from larval salivary glands (SG), imaginal discs (ID), and foregut imaginal rings (IR) were obtained by a Zeiss LSM 880 confocal microscope. An ImageJ software with TANGO modules [2] was used for segmentation of nuclei and statistical analysis of spatial organization (Figure 1). Results: We have found that the Lamin B fills the inner space of the SG and IR nuclei and colocalizes with the SG chromosomes in the A25 mutant. The fluorescent intensity of the peripheral chromatin significantly (U-test, $\mathrm{p}<0.05$ ) decreases in this mutant in comparison with the wild type. These results demonstrate that the deletion of the CaaX domain by the A25 mutation may lead to the fragmentary detachment of the chromatin from the nuclear envelope in $\mathrm{SG}$ and IR. We also found that the A25 mutation affects the localization and volume of the nucleolus in a cell type-specific manner. The $\mathrm{K} 2 \mathrm{mu}-$ tation, which affects the Lamin B alpha-helical rod but leaves the CaaX domain intact, increases the density and decreases the volume of the chromatin in the ID nuclei (Figure 2). However, it does not detach the Lamin B from the nuclear membrane in this type of cells. This result suggests that the alpha-helical rod is directly or indirectly connected to lamin-associated domains (LADs) of the chromatin. Conclusions: Our study demonstrates that Lamin B has a significant impact on the global chromatin architecture in fruit fly. Both tested mutations can cause detachments of chromatin from the nuclear periphery via different mechanisms: by redistribution of Lamin B from the nuclear envelope to the chromatin in the A25 mutant and by ceasing interactions between Lamin B and chromatin in the K2 mutant. Importantly, the lamin dysfunction has cell type-specific effects on the spatial organization of chromatin and nucleolus.

This work was supported by the NSF grant MCB-1715207 to IVS.

References: 1. Lyakhovetsky R, Gruenbaum Y: Studying lamins in invertebrate models. Adv Exp Med Biol 2014, 773:245-262. 2. Ollion J, Cochennec J, Loll F, Escude C, Boudier T: TANGO: a generic tool for highthroughput $3 \mathrm{D}$ image analysis for studying nuclear organization. Bioinformatics 2013, 29(14):1840-1841. 